\title{
CONTROLE QUÍMICO DA FERRUGEM DO CAFEEIRO (Coffea arabica L.) E SEUS EFEITOS NA PRODUÇÃO E PRESERVAÇÃO DO ENFOLHAMENTO ${ }^{1}$
}

\author{
Practices of coffee rust control related to yield and leaves retention
}

\author{
Rodrigo Luz da Cunha², Antônio Nazareno Guimarães Mendes ${ }^{3}$, Sára Maria Chalfoun ${ }^{4}$
}

\begin{abstract}
RESUMO
Objetivou-se com este trabalho um melhor ajuste do controle da ferrugem do cafeeiro (Coffea arabica L.) durante a fase de pré-colheita e verificar os seus efeitos sobre a produção e preservação do enfolhamento. $\mathrm{O}$ trabalho foi desenvolvido em uma lavoura da cultivar Acaiá Cerrado MG 1474, com seis anos de idade, no espaçamento adensado de 2,0 x 0,6 m, na qual foram testados fungicidas preventivos à base de cobre (oxicloreto de cobre e sulfato de cobre) aplicados isoladamente e associados ao sistêmico epoxiconazole; também foi utilizado o produto sistêmico aplicado isoladamente. Os cúpricos aplicados preventivamente, isoladamente ou associados ao epoxiconazole foram eficientes no controle da ferrugem, quando a incidência da doença era baixa, preservando o enfolhamento e proporcionando boa produtividade.
\end{abstract}

Termos para indexação: Coffea arabica L., ferrugem, fungicidas, produção, enfolhamento.

\begin{abstract}
This study was carried out to evaluate methods of rust control, during pre-harvesting phase, and to verify plant yield and leaves retention. Coffee cultivar used was a 6-year-old Acaiá MG 1474, with 2.0 x 0.6 meters row spacing. Copper oxichloride and copper sulphate were applied alone and associated to the systemic fungicide epoxiconazole to be tested as preventive. Copper based fungicides applied preventively or associated to the systemic epoxiconazole were efficient to rust control when applied at low disease incidences, resulting in coffee leaf preservation and adequate yield.
\end{abstract}

Index terms: Coffea arabica L., rust, fungicides, yield, foliation.

(Recebido para publicação em 26 de novembro de 2003 e aprovado em 3 de agosto de 2004)

\section{INTRODUÇÃO}

A ferrugem do cafeeiro (Coffea arabica L.) constitui ainda um fator limitante na cultura do café, sendo uma doença foliar causadora de desfolha, comprometendo a produção futura. Várias medidas de controle podem ser utilizadas no controle da ferrugem, visando sempre a aliar o sucesso no controle da doença aos aspectos de segurança ambiental e do trabalhador.

Entre as opções no controle, o emprego de fungicidas cúpricos vem sendo utilizado desde a identificação da ferrugem na Índia, Indonésia, África e no Brasil (MAYNE et al., 1933; RAYNER, 1961; MARIOTTO et al., 1976). O efeito tônico às plantas pelos cúpricos traduz-se na cor verde escura das folhas e retardamento da desfolha. Na ausência de pulverização cúprica, observa-se intensa queda de folhas na estação seca (GRIFFITHS, 1972). Todavia, dependendo do regime de chuvas, em determinados anos, torna-se difícil a execução de um programa preventivo de controle da doença, o que permite uma rápida e intensa elevação no índice de ferrugem após um período de chuvas (ZAMBOLIM et al., 1985; CHALFOUN et al., 2001). Com o advento dos fungicidas sistêmicos, parte dos cafeicultores os tem utilizado em substituição aos cúpricos. A superioridade dos sistêmicos ocorre devido às suas propriedades de absorção, translocação e modo de ação no controle da ferrugem, culminando com a diminuição de número de aplicações e diminuição da interferência de fatores climáticos nos programas de pulverizações (CARNEIRO FILHO e ISHIZAKA, 1990; MATIELLO et al., 2002). Além do efeito protetor, os sistêmicos são também curativos e erradicantes, permitindo, em alguns casos, a sua aplicação com índices de ferrugem mais elevados (MATIELLO et al., 1995; BARBOSA et al., 1998). Em situações em que a lavoura apresenta mais de 5\% de incidência de ferrugem em anos de carga elevada (MIGUEL et al., 1977; CARVALHO, 1991), tratamentos com epoxiconazole tiveram menor eficiência no controle, acentuando a bienalidade (TASSARA et al., 1998; ALMEIDA et al., 1998; ZAMBOLIM et al., 2002).

1. Parte da tese apresenta à Universidade Federal de Lavras/UFLA - Caixa Postal 3037 - 37200-000 - Lavras, MG, pelo primeiro autor, para a obtenção do título de Doutor área de concentração Fitotecnia.

2. Engenheiro Agrônomo, Doutorando/Pesquisador da Empresa de Pesquisa Agropecuária de Minas Gerais, Caixa Postal 176, $37200-000$ - Lavras, MG. Bolsista FAPEMIG. rlc@ufla.br.

3. Engenheiro Agrônomo, Dr., Professor do Departamento de Agricultura/UFLA.

4. Engenheira Agrônoma, Dra., Pesquisadora da Empresa de Pesquisa Agropecuária de Minas Gerais. 


\section{MATERIAL E MÉTODOS}

O ensaio foi conduzido no período de dezembro de 2000 a outubro de 2002 em uma lavoura de café (Coffea arabica L.) localizada no Campus da UFLA, Lavras, MG, cultivar Acaiá Cerrado, linhagem MG1474, com seis anos de idade, no espaçamento adensado de $2,0 \times 0,6 \mathrm{~m}(8.333 \mathrm{pl} / \mathrm{ha})$ e apresentando-se expectativa de boa produção.

$\mathrm{O}$ delineamento experimental utilizado foi em blocos casualizados, com seis tratamentos e quatro repetições, considerando um experimento balanceado. Cada parcela foi constituída de três fileiras de plantas com oito plantas em cada linha, sendo as seis plantas centrais consideradas úteis. $\mathrm{O}$ ano foi considerado no modelo como efeito de tempo.

O detalhamento dos tratamentos para o ensaio foram os seguintes: 1) Testemunha, sem aplicação de fontes de Cobre e fungicidas sistêmicos; 2) Oxicloreto de Cobre (3 Kg/ha), quatro aplicações, com intervalo de 30 dias, janeiro, fevereiro, março e abril; 3) Calda Viçosa Comercial, fornecendo $0,5 \mathrm{~kg} / \mathrm{ha}$ de Sulfato de Cobre, com número de aplicações e intervalo igual ao tratamento anterior; 4) Epoxiconazole $(0,6 \mathrm{l} / \mathrm{ha})$, aplicado quando a ferrugem atingiu $5 \%$ de incidência e, 60 dias após, uma aplicação de Oxicloreto de Cobre (3kg/ha); 5) Duas aplicações de Epoxiconazole, com intervalo de 60 dias; primeira com 0,6 l/ha e a segunda $0,4 \mathrm{l} / \mathrm{ha}$, com início das aplicações adotando o mesmo critério do tratamento anterior e 6) Aplicação de Epoxiconazole (0,6 l/ha), com início em janeiro, independente da incidência de ferrugem, e 60 dias após, uma aplicação de Oxicloreto de Cobre (3kg/ha).

Foram empregados como Calda Viçosa Comercial e Epoxiconazole, descrito nos tratamentos, os produtos comerciais Viça-Café (5Kg/ha) e Opus, respectivamente. Utilizou-se pulverizador costal motorizado, com gasto médio de 700 litros/ha/aplicação. As aplicações de corretivos e fertilizantes seguiram as recomendações da CFSEMG (1999). Exceção apenas para o Tratamento 3 , com relação às adubações foliares, por conter em sua formulação fontes de boro e zinco, ficando ausente essas aplicações.

Avaliaram-se, quinzenalmente, a incidência e a severidade da ferrugem a partir do início de dezembro de 2000 até o término em dezembro de 2002, observando-se os sintomas em 16 folhas por planta, no terço médio, ao acaso, do $3^{\circ}$ até o $4^{\circ}$ par de folhas, totalizando 96 folhas, em 6 plantas, por parcela útil, sendo registrada a porcentagem de folhas com ferru- gem. A severidade foi avaliada pela determinação do número médio de lesões por folha, obtido pela contagem do número total de pústulas esporuladas dividido pelo número de folhas contendo essas lesões.

Os resultados referentes à incidência e severidade da ferrugem foram utilizados para se obter o cálculo da área abaixo da curva de progresso da incidência (AACPI) e severidade (AACPS) da ferrugem, de acordo com a equação proposta por Campbell e Madden (1990).

$$
\mathrm{AACPD}=\sum_{i=1}^{n-1}\left(\frac{\mathrm{y}_{\mathrm{i}}+\mathrm{y}_{\mathrm{i}+1}}{2}\right) \cdot\left(\mathrm{t}_{\mathrm{i}+1}-\mathrm{t}_{\mathrm{i}}\right)
$$

Em que: AACPD = área abaixo da curva de progresso da doença; $y_{i}=$ proporção da doença na i-ésima observação; $t_{i}=$ tempo em dias na i-ésima observação e $\mathrm{n}=$ número total de observações.

Avaliou-se, também, a produção de café beneficiado (sacas de $60 \mathrm{~kg}$ de café beneficiado/ha) correspondente aos anos-safra 2001/2002 e 2002/2003, obtida a partir do peso de café "da roça" colhido na parcela, considerando um rendimento médio de $20 \%$ em peso para todo o experimento (MENDES, 1941).

A porcentagem de desfolha foi avaliada em todas as parcelas após a colheita, tomando-se quatro ramos produtivos por planta, registrando-se o número de pares de folhas que desprenderam no intervalo entre 10 internódios a partir do meristema apical para a base.

A análise de variância foi realizada de acordo com o modelo usual para blocos casualizados, para as variáveis área abaixo da curva de progresso da incidência (AACPI) e severidade (AACPS) da ferrugem, produção e porcentagem de desfolha. Para a comparação entre as médias dos diferentes tratamentos, efetuou-se o teste de Scott Knott ao nível de 5\% de probabilidade, utilizando-se o software SISVAR (FERREIRA, 2000).

\section{RESULTADOS E DISCUSSÃO}

A avaliação da incidência da ferrugem do cafeeiro variou com a intensidade da doença e tratamentos durante o período estudado. Em 2001, a curva de progresso da ferrugem, representada pela testemunha, teve início em fevereiro, com pico máximo no início de junho, atingindo valores superiores a $82 \%$ de incidência e prolongando-se nesse patamar até o final de agosto, após o qual sobrevieram as desfolhas. No ano seguinte, houve uma antecipação do início da curva de progresso da ferrugem para janeiro e aumento gradativo, chegando 
em maio com 37\% de incidência da doença e estendendo-se até o final do mês de agosto com $41 \%$ e, em seguida, a taxa de progresso negativa foi observada. Nesse período, a incidência da ferrugem foi menor em relação ao período anterior devido à menor produção (Figura 1). Ficou evidente que a manutenção de índices elevados de incidência de ferrugem de maio a agosto representa uma alteração no comportamento da doença devido a variações da temperatura média, à ocorrência de chuvas esporádicas durante os períodos de outono/inverno e às condições favoráveis do hospedeiro à infecção, possibilitando o prolongamento do ciclo da ferrugem durante o ano.

Os tratamentos com cúpricos e/ou sistêmicos reduziram a incidência da doença (porcentagem de folhas doentes), ficando, no período avaliado, abaixo de $35 \%$, enquanto a testemunha atingiu valores superiores a $80 \%$ nos meses de maior incidência, que foram de maio a agosto.
O resultado da análise de variância dos dados relativos à área abaixo da curva de progresso da incidência e severidade da ferrugem, produção em sacas de $60 \mathrm{~kg}$ de café beneficiado/ha e desfolha referente ao período de janeiro de 2001 a dezembro de 2002 estão apresentados na Tabela 1. Houve efeito significativo da interação Tratamento*ano sobre as quatro características estudadas e do fator isolado Tratamento sobre a área abaixo da curva de progresso da incidência e severidade da ferrugem e desfolha. Esses resultados demonstraram que os tratamentos químicos empregados afetam a curva de progresso da doença, alterando o patossistema e as demais características avaliadas.

O desdobramento da interação Tratamento*Ano referente a essas características avaliadas foram comparados pelos valores médios e representados na Figura 2.

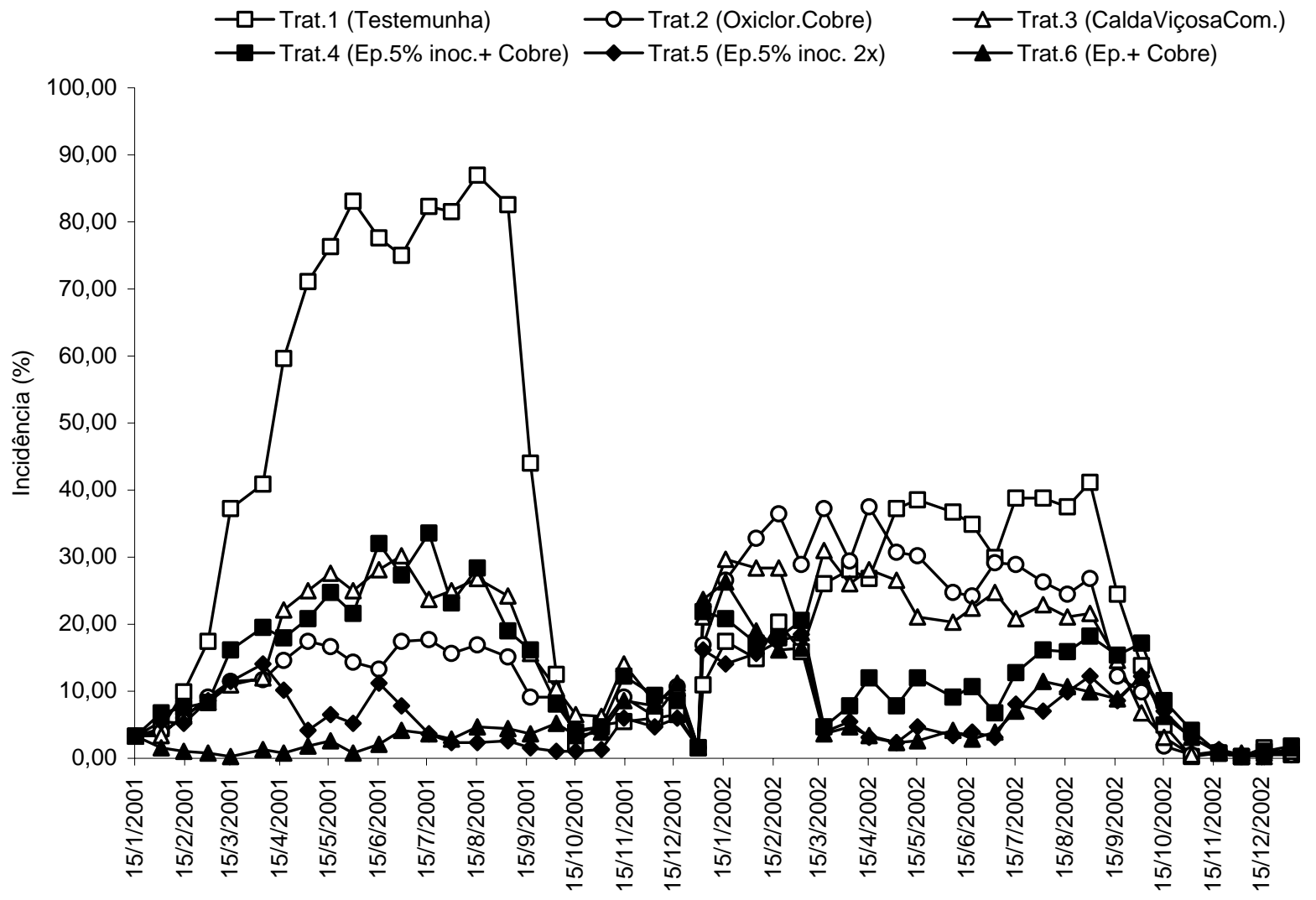

FIGURA 1 - Curvas de progresso da incidência de ferrugem do cafeeiro em lavoura submetida a diferentes tratamentos químicos nos anos de 2001 e 2002. UFLA, Lavras, MG, 2003. 
TABELA 1 - Resumo da análise de variância, médias e coeficiente de variação sobre a área abaixo da curva de progresso da incidência (AACPI) e severidade (AACPS) da ferrugem, produção em sacas de $60 \mathrm{~kg}$ de café beneficiado/ha e desfolha em porcentagem em cafeeiros submetidos a diferentes tratamentos químicos nos anos de 2001 e 2002. UFLA, Lavras - MG, 2003.

\begin{tabular}{cccccc}
\hline \multirow{2}{*}{ Fontes de variação } & \multirow{2}{*}{ G.L. } & \multicolumn{5}{c}{ Quadrado Médio } \\
\cline { 3 - 6 } & & AACPI & AACPS & Produção & Desfolha \\
\hline Tratam & 5 & $92035446,45^{* *}$ & $928994,74^{* *}$ & 333,38 & $757,60^{* *}$ \\
Blocos & 3 & 2207221,57 & 8708,56 & 40,45 & 378,46 \\
Resíduo 1 & 15 & 1099171,23 & 10139,86 & 177,28 & 163,05 \\
\hline Ano & 1 & 539243,20 & $333650,07 *$ & $30693,96 *$ & $1806,13^{*}$ \\
Resíduo 2 & 3 & 4037507,70 & 18404,26 & 153,62 & 156,78 \\
Ano * tratam & 5 & $25363265,22^{* *}$ & $392268,95 * *$ & $2504,75^{* *}$ & $716,82^{* *}$ \\
Resíduo 3 & 18 & 2166047,76 & 22803,15 & 221,20 & 96,75 \\
\hline Média geral & & 5266,15 & 630,55 & 75,78 & 45,09 \\
CV 1 (\%) & & 19,91 & 15,97 & 17,57 & 28,32 \\
CV 2 (\%) & & 38,16 & 21,51 & 16,35 & 27,77 \\
CV 3 (\%) & & 27,95 & 23,95 & 19,62 & 21,81 \\
\hline
\end{tabular}

* e **: significativo ao nível de $5 \%$ e $1 \%$ de probabilidade pelo Teste de $\mathrm{F}$, respectivamente.
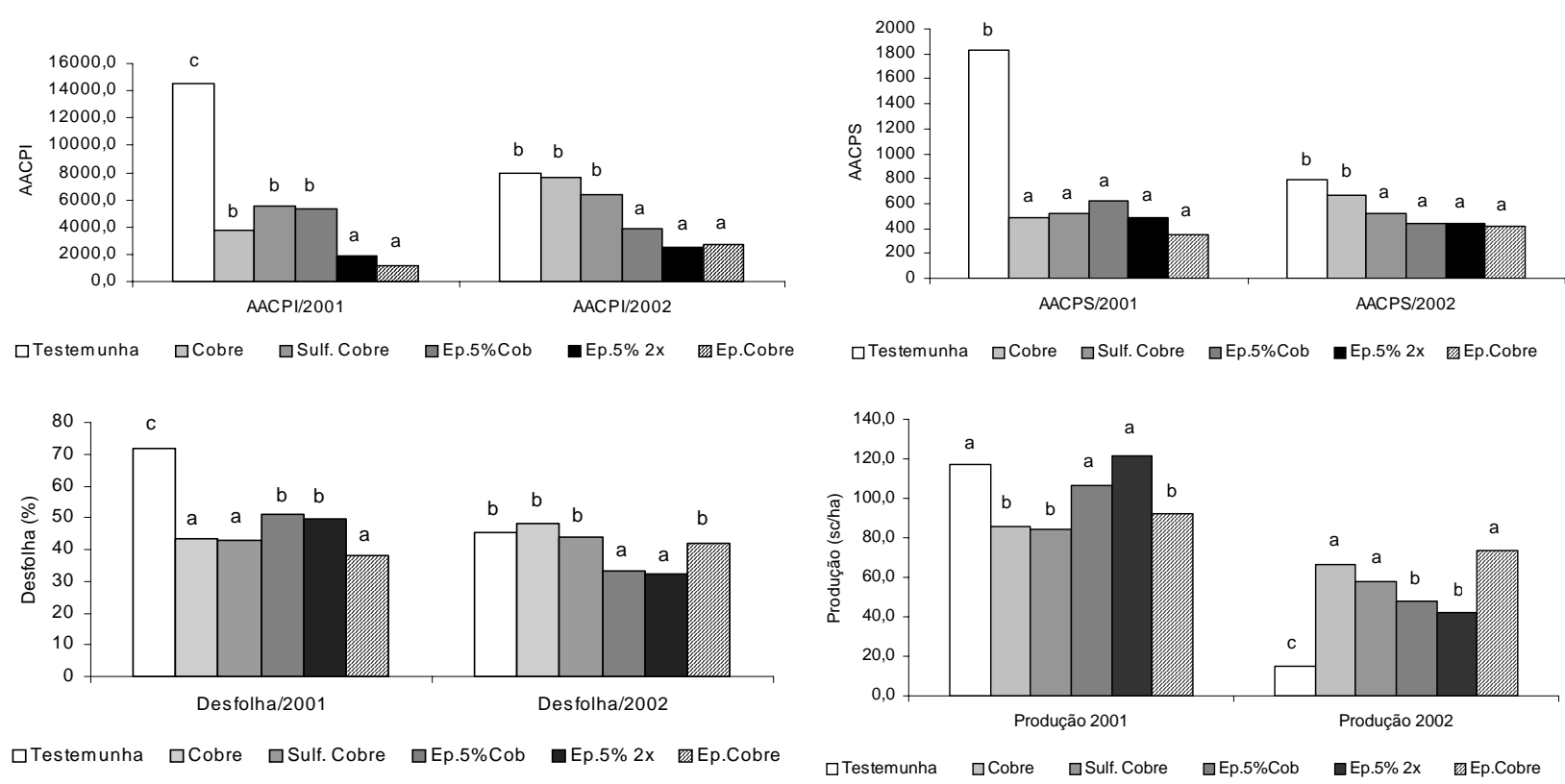

Grupo de colunas com as mesmas letras não diferem entre si pelo Teste de Scott-Knott ao nível de significância de 5\%.

FIGURA 2 - Valores médios da área abaixo da curva de progresso da incidência e severidade de ferrugem, produção (sc/ha) e desfolha em cafeeiros submetidos a diferentes tratamentos químicos nos anos de 2001 e 2002. UFLA, Lavras - MG, 2003. 
Com relação à incidência de ferrugem, observouse uma superioridade do fungicida sistêmico, embora em relação à severidade, os tratamentos tenham se equiparado quanto à área abaixo da curva de progresso de severidade (AACPS) de ferrugem (Figura 2).

No controle da ferrugem, verificou-se no ano de 2001 que os tratamentos com fungicida sistêmico associado ou não a cúprico foram mais eficientes no controle da doença, exceção apenas para o tratamento Epoxiconazole $5 \%$ + Cobre, que apresentou resultado intermediário, semelhante aos cúpricos. Tal fato justifica-se considerando as condições favoráveis do hospedeiro com alta carga pendente presente, com produção média geral de 101 sacas/ha na safra de 2001/2002, inferindose que mesmo a aplicação de um produto sistêmico no início da evolução da doença (5\%), complementado pelo fungicida cúprico, proporcionou uma certa elevação no índice da doença durante o ciclo. Nessas condições, um melhor controle foi obtido por meio do tratamento exclusivamente com o sistêmico (tratamento 5).

Observou-se, de modo geral, que no ano de 2001, o melhor controle da ferrugem e melhor preservação do enfolhamento do cafeeiro foram obtidos para os tratamentos que empregaram produtos à base de cobre, isoladamente ou associados aos sistêmicos, quando essa aplicação iniciou-se em janeiro de 2001 (tratamentos 2, 3 e 6), refletindo maiores rendimentos na safra seguinte (2002/2003). Esses resultados corroboram os de Griffiths (1972), que observou melhor enfolhamento do cafeeiro durante a estação seca em plantas que foram pulverizadas por produtos à base de cobre.

Dessa forma, os danos causados pela ferrugem devem ser evitados utilizando-se esquemas que envolvam a seguinte integração de medidas preventivas visando a atenuar a incidência da doença: o monitoramento; a seleção de fungicidas menos tóxicos ao ambiente de cultivo e aplicadores; a possibilidade de controle simultâneo de mais de uma doença, inclusive as de caráter não infeccioso (deficiências e/ou desequilíbrios nutricionais).

Na prática, tem-se, com freqüência, a adoção de medidas de controle quando as lavouras apresentam de 5 a $20 \%$ de incidência de ferrugem. Em alguns trabalhos, como os de Tassara et al. (1998), Almeida et al. (1998) e Zambolim et al. (2002) verificam-se que nessa situação tratamentos com o Epoxiconazole proporcionaram índices de controle inferior ao calendário de aplicação. Esse fato explica por que não se deve aguardar índices altos de incidência de ferrugem em anos de alta carga e em condições de clima favorável à doença, para que seja iniciado o controle com fungicidas sistêmicos.
No segundo ano de avaliação, os tratamentos que receberam os produtos à base de cobre tiveram um comportamento semelhante à testemunha. Esse fato pode ser explicado pela menor produção em relação ao ano anterior, com média geral de 50,5 sacas/ha na safra 2002/2003, acentuando a bienalidade, especialmente para a testemunha, que apresentou alta incidência de ferrugem no ano anterior (Figura 2).

Em resumo, no ano de 2001, de carga elevada, os tratamentos 5 e 6 nos quais utilizou-se o produto sistêmico epoxiconazole foram mais eficientes, conforme previsto. No ano de 2002, de carga média, os mesmos tratamentos, incluindo o tratamento 4, ainda foram os mais eficientes, fato atribuído à elevação antecipada dos índices de ferrugem (ponto de inflexão da curva no mês de janeiro). Dessa forma, os tratamentos 2 e 3, com fungicidas cúpricos, cujas épocas de início foram préfixadas, tiveram sua eficiência negativamente afetada, o que concorda com as recomendações de que essa classe de fungicidas deve ser aplicada na ausência de sinais visíveis da doença.

Analisando as produções em relação aos tratamentos, nas duas safras (Figura 2), observa-se que os tratamentos que mantiveram o nível de ferrugem sob controle proporcionaram maiores rendimentos. Todavia, o nível de produção e a incidência da doença na lavoura é um fator importante a determinar maior ou menor grau de incidência da ferrugem, concordando com os estudos relacionados a essa característica (MIGUEL et al., 1975; CARVALHO, 1991). A safra de 2002/2003 reflete os efeitos dos tratamentos utilizados no ano anterior, com destaque para o rendimento dos tratamentos com epoxiconazole e oxicloreto de cobre (tratamento 6), oxicloreto de cobre (tratamento 2) e sulfato de cobre (tratamento 3). Houve efeito, também, da bienalidade, principalmente para os tratamentos epoxiconazole com aplicação em 5\% de incidência de ferrugem e cobre (tratamento 4), epoxiconazole $5 \%$ em duas aplicações (tratamento 5) e a testemunha, sem controle, acentuando consideravelmente a redução na produção, principalmente no segundo ano, com 14,7 sc/ha (tratamento 1), evidenciando a importância do controle da ferrugem.

A desfolha foi avaliada após a colheita, nas duas safras. Essa característica é função de várias causas de ordem fisiológica, biológica, química, física, além de outras. No presente estudo, as principais causas da desfolha estão sendo atribuídas à natureza biológica, pela ação da alta incidência e severidade da ferrugem, além da ação física pela colheita, não desprezando as outras 
causas que podem estar agindo simultaneamente, provavelmente em menor intensidade.

Após a avaliação da desfolha na safra de 2001/2002, verificaram-se os efeitos benéficos na preservação do enfolhamento das plantas nos tratamentos 2, 3 e 6, que continham em sua formulação o micronutriente cobre, sendo esses resultados superiores aos tratamentos 4 e 5, que levaram produtos sistêmicos aplicados com 5\% de incidência de ferrugem. Apesar de os tratamentos 4 e 5 com produtos sistêmicos mostraremse eficientes no controle da ferrugem, foram inferiores na preservação do enfolhamento, quando comparados aos tratamentos com produtos cúpricos.

Algumas características avaliadas mostraram resultados em comum, principalmente na incidência de ferrugem, produção e desfolha. Da mesma forma em que ocorreu maior intensidade das doenças e maior produção no ano de 2001, a desfolha foi também significativamente maior nesse ano, registrando uma porcentagem média de 49\% em relação a 2002, com 40\% de desfolha.

A testemunha, sem controle, apresentou mais de $70 \%$ de desfolha, deixando a planta praticamente sem folhas após a colheita de 2001 e, conseqüentemente, comprometendo a produção do ano de 2002, conforme verificado na Figura 2.

\section{CONCLUSÕES}

Medidas de controle com fungicidas cúpricos, tais como o oxicloreto de cobre e calda viçosa comercial, aplicados preventivamente ou associados com o produto sistêmico epoxiconazole, são eficientes no controle da ferrugem, se aplicados quando a incidência da doença é baixa, preservando o enfolhamento e, conseqüentemente, proporcionando bons índices de produtividade.

\section{REFERÊNCIAS BIBLIOGRÁFICAS}

ALMEIDA, S. R.; MATIELLO, J. B.; FERREIRA, R. A. Efeito de dose, número de aplicações e adição de cobre ou espalhante na ação do fungicida Opus (Epoxiconazole) no controle curativo da ferrugem do cafeeiro. In: CONGRESSO BRASILEIRO DE PESQUISAS CAFEEIRAS, 24., 1998, Rio de Janeiro. Resumos... Rio de Janeiro: PROCAFÉ, 1998. p. 49-51.

BARBOSA, J. C.; MENDONÇA, P. L. P. de; BEGLIOMINI, E.; TOLEDO, V. J. G. Compatibilidade de opus (epoxiconazole) com os adubos foliares da basf em relação ao controle da ferrugem do cafeeiro - Hemileia vastatrix Berk e Br. In: CONGRESSO BRASILEIRO DE PESQUISAS CAFEEIRAS, 24., 1998, Poços de Caldas.
Trabalhos apresentados... Poços de Caldas: PROCAFÉ, 1998. p. 112.

CAMPBELL, C. L.; MADDEN, L. V. Introduction to plant disease epidemiology. New York: John Wiley \& Sons, 1990. 655 p.

CARNEIRO FILHO, F.; ISHIZAKA, A. M. Controle da ferrugem do cafeeiro com diversos fungicidas protetores, sistêmicos e em mistura com inseticidas em aplicações no solo e foliar. In: CONGRESSO BRASILEIRO DE PESQUISAS CAFEEIRAS, 16., 1990, Espírito Santo do Pinhal. Resumos... Rio de Janeiro: IBC, 1990. p. 98-99.

CARVALHO, V. L. de. Influência de níveis de produção sobre a evolução da ferrugem e a composição química das folhas do cafeeiro. 1991. 85 f. Dissertação (Mestrado em Fitopatologia) - Universidade Federal de Lavras, Lavras, 1991.

CHALFOUN, S. M.; CARVALHO, V. L. de; PEREIRA, M. C. Efeito de alterações climáticas sobre o progresso da ferrugem (Hemileia vastratrix Berk. \& Br.) do cafeeiro (Coffea arabica L.). Ciência e Agrotecnologia, Lavras, v. 25, n. 5, p. 1248-1252, set./out. 2001.

COMISSÃO DE FERTILIDADE DE SOLO DO ESTADO DE MINAS GERAIS. Recomendações para o uso de corretivos e fertilizantes em Minas Gerais: $5^{a}$ aproximação. Lavras, 1999. 359 p.

FERREIRA, D. F. Análises estatísticas por meio do SISVAR para Windows: versão 4.0. In: REUNIÃO ANUAL DA REGIÃO BRASILEIRA DA SOCIEDADE INTERNACIONAL DE BIOMETRIA, 45., 2000, São Carlos, SP. Resumos... São Carlos: UFSCar, 2000. p. 235.

GRIFFITHS, E. Negative effects of fungicides. Tropical Science, London, v. 14, n. 1, p. 79-89, Mar. 1972.

MARIOTTO, P. R.; FIGUEIREDO, P.; SILVEIRA, A. P.; JÚNIOR, G.; ARRUDA, H. V.; LOPES, H.; OLIVEIRA, E. G.; FERREIRA JÚNIOR, B. L.; OLIVEIRA FILHO, N. L. Estudos sobre o controle químico da ferrugem do cafeeiro (Hemileia vastatrix Berk \& Br.) e seus efeitos na produção, nas condições do estado de São Paulo. O Biológico, [S.l.], v. 45, n. 910, p. 165-174, 1976. 
MATIELLO, J. B.; ALMEIDA, S. R.; FERREIRA, R. A. Comparação de fungicidas sistêmicos no controle da ferrugem do cafeeiro sob condição de alta infecção. In: CONGRESSO BRASILEIRO DE PESQUISAS CAFEEIRAS, 21., 1995, Caxambu. Resumos... Caxambu: PROCAFE, 1995. p. 21-22.

MATIELLO, J. B.; SANTINATO, R.; GARCIA, A. W. R.; ALMEIDA, S. R.; FERNANDES, D. R. Cultura de café no Brasil: novo manual de recomendações. Rio de Janeiro: MAPA/PROCAFÉ, 2002. 387 p.

MAYNE, W. W.; NARASIMHAN, M. J.; SREENIVASAN, M. S. Spraying of coffee in South India. Mysore Coffee Exp Statn. BulI, [S.l.], n. 9, p. 69, 1933.

MENDES, J. E. T. Melhoramento de Coffea arabica L. var. bourbon. Bragantia, Campinas, v. 1, n. 1, p. 3-35, jan. 1941.

MIGUEL, A. E.; MANSK, Z.; MATIELLO, J. B.; ALMEIDA, S. R. Efeitos de fungicidas no controle de Cercospora coffeicola em frutos de café. In: CONGRESSO BRASILEIRO DE PESQUISAS
CAFEEIRAS, 3., 1975, Curitiba. Resumos... Rio de Janeiro: IBC-GERCA, 1975. p. 58-61.

RAYNER, R. W. Germination and penetration studies on coffee rust (Hemileia vastatrix Berk. \& Br.). Annual of Applied Biology, Warnick, v. 49, n. 3, p. 497-505, 1961.

TASSARA, D.; SANTINATO, R.; MENDONÇA, P. L. P.; ANTÔNIO, A. M. D.; PEREIRA, E. M. Programa de controle da ferrugem do cafeeiro com fungicida sistêmico opus (epoxiconazole) isoladamente e em combinações com cobox ou hidróxido basf. In: CONGRESSO BRASILEIRO DE PESQUISAS CAFEEIRAS, 24., 1998, Poços de Caldas. Trabalhos apresentados... Poços de Caldas: PROCAFÉ, 1998. p. 210-211.

ZAMBOLIM, L.; MARTINS, M. C. del P.; CHAVES, G. M. Café. Informe Agropecuário, Belo Horizonte, v. 11, n. 131, p. 64-75, nov. 1985.

ZAMBOLIM, L.; VALE, F. X. R. do; COSTA, H.; PEREIRA, A. A.; CHAVES, G. M. Epidemiologia e controle integrado da ferrugem-do-cafeeiro. In: ZAMBOLIM, L. (Ed.). O estado da arte de tecnologias na produção de café. Viçosa: UFV, 2002. cap. 10, p. 369-450. 